\title{
Editorial: City-Wide Sanitation: The Urban Sustainability Challenge
}

\author{
Christoph Lüthi ${ }^{1 *}$, Juliet Willetts ${ }^{2}$ and Sabine Hoffmann ${ }^{3}$ \\ ${ }^{1}$ Department of Sanitation, Water and Solid Waste for Development (Sandec), Swiss Federal Institute of Aquatic Science and \\ Technology, Dübendorf, Switzerland, ${ }^{2}$ Institute for Sustainable Futures (ISF), University of Technology Sydney, Sydney, \\ NSW, Australia, ${ }^{3}$ Department of Environmental Social Sciences (ESS), Swiss Federal Institute of Aquatic Science and \\ Technology, Dübendorf, Switzerland
}

Keywords: non-sewered sanitary systems, equity, sdg \#6, urban sanitation, fecal sludge management (FSM)

\section{Editorial on the Research Topic}

\section{City-Wide Sanitation: The Urban Sustainability Challenge}

The Sustainable Development Goals (SDGs) adopted in 2015 have led to a paradigm shift in how urban sanitation is managed. Targets 6.2 (safely managed sanitation and hygiene services) and 6.3 (reducing the portion of untreated wastewater) now put the focus on managing the entire sanitation chain, encompassing containment, emptying, transport, treatment, and safe reuse or disposal. This has major implications for urban areas, which are a major contributor of untreated wastewater, creating hotspots for environmental degradation and public health hazards (both within and outside of cities) impairing social and economic productivity.

While national water, sanitation and hygiene (WASH) targets increasingly reflect SDG ambitions, aiming to provide universal coverage and reach higher levels of service, this is proving difficult in the urban context. Recent Joint Monitoring Program (JMP) and Global Analysis and Assessment of Sanitation and Drinking-Water (GLAAS) reports have underlined uneven progress in sanitation coverage, with progress disproportionally benefiting the wealthy, leaving the urban poor unserved (JMP 2019 update). According to the Joint Monitoring Programme, the gap between the richest and poorest has been reduced in 52 countries but increased in 22 countries-mostly countries emerging from conflict (UNICEF/WHO, 2019, p. 34).

\section{THE URBAN CHALLENGE}

Cranfield University, United Kingdom

*Correspondence: Christoph Lüthi luethi@eawag.ch

Specialty section:

This article was submitted to Water and Wastewater Management,

a section of the journal

Frontiers in Environmental Science

Received: 20 July 2020

Accepted: 09 September 2020

Published: 19 October 2020

Citation:

Lüthi C, Willetts J and Hoffmann S (2020) Editorial: City-Wide Sanitation:

Most cities in low and middle-income countries are growing bigger and denser, with vast underserved informal and peri-urban settlements. To achieve the SDG "urban" goals and provide a citywide solution to sanitation, a more integrated and inclusive approach is needed to cover all urban areas. This novel concept supports a blended approach that includes a menu of solutions such as onsite sanitation systems with fecal sludge management (FSM), decentralized or small-scale systems for areas too far from existing sewers or too dense for household solutions and, where this makes sense, piped sewers (e.g., central business districts). Total sanitation coverage for cities in low and middle-income countries will need to comprise a mix of different contextualized solutions. This special edition of Frontiers in Environmental Science provides a deeper insight into the institutional, technological, and socio-economic challenges of the new urban sanitation paradigm.

Equitable services for all urban dwellers are at the core of citywide inclusive sanitation (CWIS). While the exact definition of CWIS is still evolving, the guiding principles first published in 2016 (Citywide Inclusive Sanitation: A Call to Action: https://citywideinclusivesanitation. $\mathrm{com} /$ ) have since gathered momentum with development partners, governments and service providers in many countries. CWIS thinking rests on four principles: (i) Prioritize the human right of citizens to sanitation-equitable and accessible for all; (ii) Deliver safe management along the whole sanitation service chain, from the toilet to safe treatment and reuse; (iii) 
Integrate sanitation in urban planning and renewal, providing liveable and sanitary environments; and (iv) Commit to working in partnership to deliver citywide inclusive sanitation, including formal and informal partners.

While CWIS is still an evolving framework for informing urban sanitation investment programming, Citywide Inclusive Sanitation has already started to shape programming and investments from development banks and major sector actors.

This special Research Topic of Frontiers in Environmental Science is the first collection of academic contributions that seek to conceptualize and frame the new citywide inclusive sanitation paradigm for urban sanitation in low- and middle income countries. We seek to critically evaluate existing alternative approaches to urban sanitation, introducing new citywide equitable sanitation concepts and solutions. In the 13 papers selected for this special edition, we provide a historical perspective on the emergence of this new approach, a definition of the main features and pillars of CWIS and we provide insights for some of the menu options that make citywide sanitation an actionable and implementable approach for the rapidly urbanizing global South. The special edition is structured in four parts: Part 1 provides an overview of the conceptual framework of citywide inclusive sanitation and defines key CWIS concepts and principles. Papers in Part 2 introduce methods and applied approaches that can help disentangle the complexities of citywide sanitation. Part 3 provides insights on fecal sludge management approaches to safely empty, transport, treat and dispose of or re-use fecal sludge. Finally, Part 4 addresses the role nonconventional small-scale or decentralized sanitation can play in providing equitable access to sustainable sanitation services.

\section{(i) CWIS Concept}

Citywide inclusive sanitation as a concept is being continuously refined through on-going debate, practice and implementation. The growing body of programmes and investments informed by the approach already provides opportunity for reflection. In addition, emerging analyses point to ways that the approach could be sharpened to address key urban sustainability issues, including public health, climate change and economic performance.

Two policy briefs provide insight into the CWIS concepts and principles employed by two significant funders of urban sanitation, with a common narrative on the need for radical change from "business as usual." Schrecongost et al. review the genesis of CWIS and lay out core outcomes of the required public service delivery system, namely equity, safety, and sustainability across all areas of a city, not just for sewered areas. They assert that this system must demonstrate three functions: a responsible authority with a clear, inclusive mandate for service delivery; a mechanism to ensure accountability for performance against this mandate; and processes for managing and planning resourcing including financing, assets and human resource. In their contribution, Gambrill et al. also assert that conventional sewerage and wastewater treatment should not be considered the only option and that a range of solutions-both on-site and sewered, centralized or decentralized-must be tailored to the realities of growing cities. The authors point out the need for changed mindsets amongst governments, development agencies and consultants, evolution of engineering curricula to include non-conventional solutions and a rethink on the way sanitation infrastructure is funded.

In their contribution, Mills et al. examine contamination, climate change and costs as three factors that require increased attention to reach key outcomes integral to inclusive citywide sanitation, namely public health, sustainability and economic performance. The authors provide available evidence on these three areas, including fecal contamination risks associated with onsite, decentralized and centralized systems in urban living environments, integration of climate change impacts such as flooding into sanitation planning, and use of cost effectiveness analysis against consistent service objectives to support improved comparison of the mix of sanitation options likely to be appropriate to different contexts across a city.

\section{ii) Methods}

Citywide inclusive sanitation as a novel approach to urban sanitation requires an array of new tools and methods to provide answers for planning and programming non-conventional sanitation solutions.

The paper by Narayan et al. investigates if social network analysis (SNA) provide a viable approach that can deal with the complexity of the set of stakeholders involved in the governance of Water, Sanitation and Hygiene (WASH) and the diversity of their interests. The SNA is applied to study the governance of decentralized wastewater treatment in four cities of India). The results corroborate key differences between mega and secondary cities in terms of institutions, community engagement and the overall sanitation situation. These findings are relevant if we are to confront the politics and institutional blockages that prevent the provision of safely managed sanitation for all.

Peal et al. describe a methodology for rapid analysis of excreta flows in urban areas using so called excreta or shit-flow diagrams (SFDs), a methodology that has gained popularity in the last few years. The authors provide the first comprehensive analysis of SFDs conducted in 39 cities with a population of 72 million and provide an insight into the main sources of unsafely managed excreta. The study helps understand sanitation failures at supracity level, ranging from non-contained fecal sludge in urban areas to wastewater that is delivered to treatment but not properly treated. The paper provides evidence of the urgent need for improved management and monitoring of urban sanitation in cities around the world.

In their contribution on "Sanitation Cityscapes," Scott and Cotton provide a new conceptual framework for citywide urban sanitation that embeds sanitation within wider urban governance. Urban systems are not linear, and the Cityscape provides a conceptual framing of how sanitation services are located with respect to urban residents' demand, tenure, neighborhood typologies (the living environment), the ways services are delivered (the service delivery environment), and the ability of the city to deliver basic services (the enabling environment). They propose 16 core indicators to describe any given sanitation service delivery context using data drawn from an application of the framework in an Ethiopian town.

Mtika and Tilley raise a number of pertinent issues regarding sanitation planning in a small town in Malawi, 
adopting the Community-Led Urban Environmental Sanitation (CLUES) approach. The 2-year field research revealed that the high turnover of government staff affected institutional knowledge retention, acceptance and continuity and ultimately the potential to engage in a successful planning exercise. Because baseline data was outdated or non-existent, data collection activities consumed unexpected amounts of time. Most importantly, stakeholder participation was limited and relied on participation and transportation payments, which have become a permanent feature of "community development" in many African countries. They therefore propose a simplified version, making use of available data and followed only by small towns with a functioning planning department, adequate tax collection, organized community groups and a budget for final implementation.

\section{iii) Fecal Sludge Management (FSM)}

In embracing onsite systems as part of the technology mix, finding solutions to safely empty, transport, treat and dispose of or re-use fecal sludge (FS) is an significant priority.

Russel et al. argue that Container-Based Sanitation (CBS) is now established as a new type of improved sanitation system for poor urban areas that provides a sustainable service for the entire sanitation service chain. The authors outline the main challenges that need to be resolved in order for CBS to reach maturity and scale, including official recognition by local authorities and utilities, improved regulation and innovative financing.

Mehta et al. report on the experience with scheduled desludging of onsite containment in urban India, which sees FSM as a regular service similar to municipal solid waste collection. The paper provides an account of emerging experience with the design and implementation of scheduled desludging to achieve social and environmental benefits in two Indian cities.

McConville et al. present a structured three-step approach for comparing nutrient-recovery FS treatment systems against a wide range of sustainability criteria covering five dimensions: (i) health, (ii) financial, (iii) social, (iv) technical, and (v) institutional. The authors apply the approach in the context of selecting the most appropriate and acceptable nutrient-recovery options for upgrading a FS treatment system in Kampala, Uganda. Used in this way, the approach provides decisionmaking support for both short-term and long-term investments with a view to deliver citywide inclusive sanitation based on locally specific prerequisites.

iv) Decentralized Sanitation

Bridging gaps between household scale and city scale, decentralized solutions hold significant promise, however their governance, regulation and management poses new challenges, explored in these three contributions.

Reymond et al. investigate the governance arrangements necessary for the successful scaling up of small-scale wastewater treatment plants (SSTPs) in low and middle-income countries, along the whole project cycle, from technology selection to operation and maintenance. Based on the analysis of the scaling up process in India, the study sought to understand why many SSTPs underperformed and identify the required governance arrangements for such systems to fulfill their potential in terms of urban sanitation coverage and water reuse, and their role in citywide inclusive sanitation. The paper explores the concept of a "hybrid governance approach" that blends hierarchical, market, and network governance to foster market regulation and stakeholder coordination and promotes the need for dedicated units at state and city scale to manage distributed systems.

Also with a focus on governance and management, Willetts et al. report on research in Indonesia, where more than 20,000 community-scale systems have been built in low-income urban communities. The study investigated the potential of a co-management approach between city governments and communities, to overcome the current struggles faced by communities unable to cope with the technical, social and financial management of these systems. The proposed comanagement approach assists city governments fulfill their legal mandate for ensuring services, and is an important advance given the increasing trend of community-scale systems in low and middle- income countries.

Tackling the critical area of regulation, Schellenberg et al. focus on wastewater discharge standards in India with a view to how these influence the place of innovative, decentralized, ecologically sound solutions, including those that enable re-use. Drawing on examples from other emerging economies and in Western Europe, the paper looks at how revised policy and regulatory approaches could benefit the fertile technology landscape in India, providing new opportunities for creative approaches to urban sanitation.

\section{A FINAL NOTE}

These 13 contributions together provide a way forward to tackle the impasse faced in many low- and middle-income country cities in improving urban sanitation services. Central to this, is the acceptance of urban sanitation as a public good that requires governance structures and investments that ensure services for all parts of a city and to all people. Within this, innovation and sustainable solutions can emerge, including at household, decentralized and centralized scales, with an eye to meeting the impending impacts of climate change, facilitating re-use of precious resources including water and nutrients, and, in line with sanitation's core objective, protecting public health.

\section{AUTHOR CONTRIBUTIONS}

All authors listed have made a substantial, direct and intellectual contribution to the work, and approved it for publication.

\section{ACKNOWLEDGMENTS}

We thank the research team of the strategic program Wings (Water and sanitation innovations for non-grid solutions), partially funded by Eawag Discretionary Funds, for conceptual support and intellectual inspiration. 


\section{REFERENCES}

UNICEF/WHO. (2019). Progress on Drinking Water, Sanitation and Hygiene 2000-2017. p. 138.

Conflict of Interest: The authors declare that the research was conducted in the absence of any commercial or financial relationships that could be construed as a potential conflict of interest.
Copyright (c) 2020 Lüthi, Willetts and Hoffmann. This is an open-access article distributed under the terms of the Creative Commons Attribution License (CC BY).

The use, distribution or reproduction in other forums is permitted, provided the original author(s) and the copyright owner(s) are credited and that the original publication in this journal is cited, in accordance with accepted academic practice. No use, distribution or reproduction is permitted which does not comply with these terms. 